\title{
An Efficient and Reliable Routing Protocol for WSNs Based on Quantum Artificial Bee Colony Algorithm
}

\author{
Huadong Wang and Dayu Wang \\ School of Computer Science and Technology, Zhoukou Normal University, \\ Zhoukou, 466001, P. R. China \\ *Correspondence author: zhoukou985211@163.com
}

\begin{abstract}
The cluster head random selection in the classical clustering LEACH algorithm on wireless sensor network does not consider the residual energy of nodes, node position and node intensity etc.. This paper proposed the efficient and reliable WSNs clustering algorithm based on the quantum artificial bee colony algorithm aiming to reduce the node energy consumption and prolong the network lifetime. The simulation indicated that the improved artificial bee colony routing clustering algorithm owns more uniform clustering than the LEACH and HEED protocol with more reasonable cluster head selection, which has prolonged the network lifetime effectively and ensures the reliability of the communications within the clusters.
\end{abstract}

Keywords: Wireless sensor network; Routing algorithm; Quantum artificial bee colony algorithm; High efficiency; Reliability

\section{Introduction}

In recent years, with the rapid development, wireless sensor network has been widely applied in the military, medicine, security and environmental monitor etc. [1]. Wireless sensor network is characterized by low cost, low speed and low power consumption. The node energy consumption is the main indicator for the life cycle of wireless sensor network. The sensor network node is usually powered by the battery. The longer of the communication distance between nodes, the larger of the energy consumption. Therefore, lots of researchers have conducted research on how to improve the network consumption and node distribution. Intelligent clustering routing algorithm has been one of the key technologies for wireless sensor network to save the network consumption and node deployment [2-3]. The low energy adaptive clustering hierarchy protocol (LEACH) is a classical routing intelligent clustering algorithm that can prolong the network lifetime to a great extent. However, this algorithm also owns certain deficiency including the following aspects:

(1) Every node in the protocol is selected as the cluster head with a certain probability. Due to the randomness of the selected probability, the distributed position of every round of the cluster head node cannot be guaranteed in the current small region. The longer communication distance from the other heads to the cluster head will bring out larger energy consumption.

(2) When selecting the cluster head, the protocol has not considered whether the residual energy of the nodes can act as the role of the cluster head. The node may only have a small amount of energy for the cluster head selection, which caused the fast death of the node because of too much energy consumption. Besides, the data gathered by the cluster head cannot be transmitted to Sink, which led to the mistake in the process of the data acquisition.

(3) When the cluster head is selected in the protocol, the other nodes will choose to join in the cluster according to the received signal strength. In general, the cluster head with 
strong signal will have wider communication area. On the contrary, the cluster head with a weak signal will have smaller communication area. The longer distance from the cluster head to the fringe node between clusters will result in huge energy consumption, while the nodes nearby the cluster head will have minimal energy consumption, which caused the unbalanced network energy consumption that will affect the network lifetime directly.

To cure the above problems, this paper presents an intelligent clustering algorithm based on the quantum artificial bee colony algorithm under the improved intelligent clustering routing reliable communication algorithm based on the LEACH protocol.

\section{The Related Work}

The flow chart of the low energy adaptive clustering hierarchy protocol (LEACH) was showed the Figure 1. Many researchers have conducted a large amount of works on the improvement of the LEACH algorithm. Though the LEACH algorithm saved the node energy and improved network efficiency in some respect, it cannot balance the network energy consumption distribution. The literature [4] carried out the improvement and optimization on the deficiencies of the LEACH algorithm to guarantee the quantity and the energy of the cluster heads so as to ascertain the proper quantity of the cluster heads. The literature [5] put forward and designed an adaptive inertia weight adjustment strategy based on the swarm particle condition and proposed an adaptive discrete particle swarm optimization algorithm to guarantee the reliability of data transmission and network balance aiming at the load unbalance between clusters as well as the unreliable communication problems. It was reported in [6] the author improved the cluster head selection mechanism of LEACH, apply the quantum particle swarm to optimize the cluster selection and then proposed a new clustering algorithm for the node load balance aiming at the limited node energy and unbalanced energy loss of different nodes. In order to accelerate the routing search speed of wireless sensor network and reduce the pathsearch energy consumption, Yisong Jiang et al [7] put forward the dynamic clustering routing algorithm on wireless sensor network based on the Best-Worst Ant System (BWAS) to slow down the energy consumption of network node and prolong the network lifetime with rather strong robustness. The literature [8] proposed a new Energy-efficient Distributed Clustering Algorithm (BPEC) by taking the ratio of average residual energy of the neighboring nodes to the residual energy of the node itself as the main parameter to compete for the cluster head and the "degree" of node as the aided parameter for the cluster head competition to achieve the balanced load and prolong the network life cycle. The literature [9] transformed the clusters as the cluster partition with the approximate optimization goal as well as the cluster head selection issue and then adopted a heuristic clustering control algorithm. This paper put forward an efficient and reliable routing algorithm on wireless sensor network based on the quantum artificial bee colony algorithm by summarizing the research results of the above researchers and combing the emerging swarm intelligence algorithm to enhance the network clustering efficiency and improve the network efficiency and reliability. 


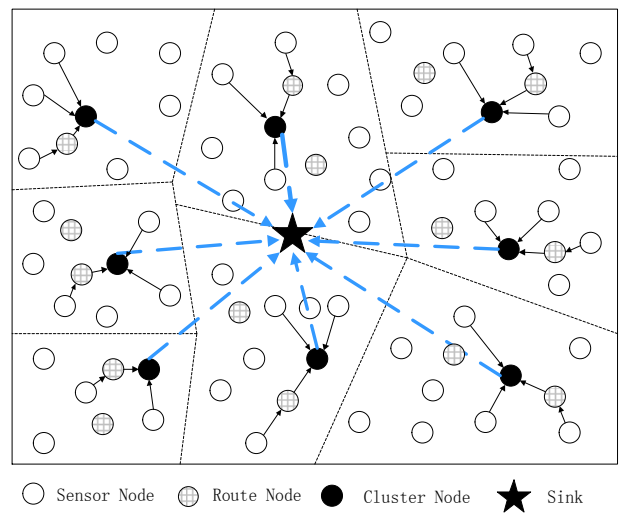

Figure 1. LEACH Routing Clustering Algorithm

\section{Problem Description}

In the application of wireless sensor network, the majority of energy of the sensor nodes is consumed on the wireless communication module. In the process of the wireless communication, the network construction, data transmission and acceptance account for the maximum energy consumption. Therefore, the network energy consumption mathematics model shall be firstly established. This paper provided the energy consumption model of transmitting a k-bit packet transceiver according to the working energy model of the sensor node in the literature [6], shows in Figure 2.

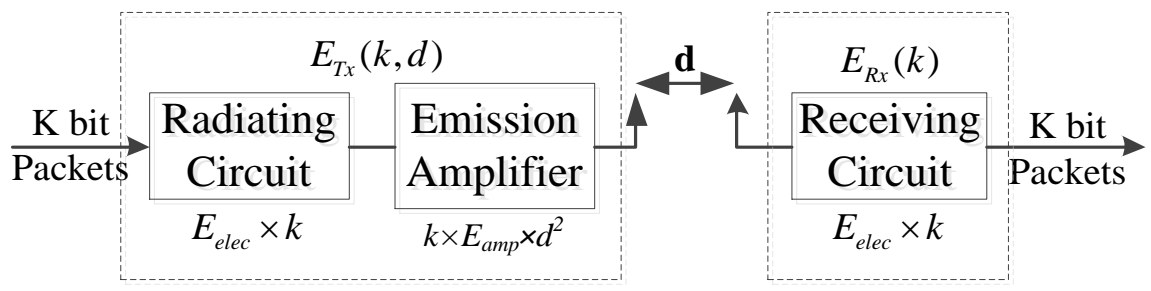

Figure 2. Energy Model of Wireless Sensor Network Communication

In Figure $2, E_{\text {elec }}$ represents wireless communication transmitting circuit and the receiving circuit, each transmit / receive 1 bit of data packets the consumption of energy, $E_{\text {amp }}$ represents an enlarged section transmitting circuit packet units required per bit energy consumed. The consumption of energy of mathematical formulas

$$
E_{T x}(k, d)=E_{\text {elec }}(k)+E_{\text {amp }}(k, d)=E_{\text {elec }} \times k+E_{\text {amp }} \times k \times d^{2}
$$

The node receives the information required energy mathematical formula:

$$
E_{R x}(k)=E_{T x}(k)=E_{\text {elec }} \times k
$$

We put the network energy consumption indicators as the evaluation of the total energy consumption of network, the network decided to network life cycle energy consumption. Networking consumption formula:

$$
E_{n e t}=\frac{\sum_{i=0}^{n} E_{r} \times n^{*}}{n^{2}}
$$


Where $n$ represents the current polling survival number of nodes, $E_{r}$ is the energy consumed by the current polling, $\sum_{i=0}^{n} E_{r}$ is the sum of the energy consumed by the packets and the received packets during the networking. $n^{*}$ is the total number of sensors, $n^{*} / n$ is the survival rate of the network nodes.

According to the proposed in literature [6], in order to reduce the overall energy consumption of the network, we find the $K$ cluster head to WSNs cluster all nodes to the square of the corresponding cluster head distance and all cluster heads to the base station of the square of the distance and smaller, objective function is

$$
\text { fitness }=\sum_{j}^{m}\left(\sum_{i}^{n_{j}} d_{i j}^{2}+b_{j}^{2}\right)
$$

Where $m$ is the number of cluster head, $n_{j}$ for the number of nodes in the $\mathrm{j}$-th cluster head, and $d_{i j}$ the $\mathrm{j}$-th cluster head to the $i$-th node distance, $b_{j}$ for the distance of the $\mathrm{j}$-th cluster head to the base station.

\section{Quantum Artificial Bee Colony Algorithm}

As the intelligent optimization algorithm to simulate the group hunting of natural creatures, the swarm intelligence algorithm has provided a new technological means for the global optimization problem--NP hard problem in the computer science, computer application and management control science fields etc.. As the research hotspot in recent years, this algorithm has been widely applied in various fields. The current emerging intelligent optimization-artificial bee colony $(\mathrm{ABC})$ is an intelligent swarm optimization algorithm to simulate the search behavior of bee forage. This algorithm has attracted the attention of wide researchers with less parametric variation, easily achieved and easy to be calculated [10].

The traditional artificial bee colony algorithm (QABC) has achieved great results in multi-function optimization, knapsack problems and travelling salesman problems etc.. However, this algorithm will show pre-mature atmosphere in the optimum process and will be easily caught in the local convergence with bad optimized effect in some respect. Therefore, this paper introduced the concept of quantum computation in the artificial bee colony algorithm, integrated the quantum computation into the artificial bee colony algorithm, put forward the quantum artificial bee colony algorithm enhanced the global search capability with favorable convergence and applied it into wireless sensor network routing optimization to improve the network efficiency and reliability.

\subsection{Quantum Position of Quantum Bee Colony}

The location of the major bee particle quantum bits to describe the vector is represented by a single qubit $[\alpha, \beta]^{\mathrm{T}}$, while $\alpha^{2}+\beta^{2}=1$, Define the position of the k-bee mathematical formula $W_{k}$ is

$$
W_{k}=\left[\begin{array}{lllll}
\alpha_{k 1} & \alpha_{k 2} & \alpha_{k 3} & \mathrm{~L} & \alpha_{k l} \\
\beta_{k 1} & \beta_{k 2} & \beta_{k 3} & \mathrm{~L} & \beta_{k l}
\end{array}\right]
$$

The evolution of individual in the colony is by colony where the location update to achieve, we define the $k$ bee location corresponding to the i-th one qubit formula for

$$
\begin{aligned}
W_{k i}^{t+1} & =a b s\left(U\left(\theta_{k i}^{t+1}\right) W_{k i}^{t}\right) \\
U\left(\theta_{k i}^{t+1}\right) & =\left[\begin{array}{ll}
\cos \theta_{k i}^{t+1} & -\sin \theta_{k i}^{t+1} \\
\sin \theta_{k i}^{t+1} & \cos \theta_{k i}^{t+1}
\end{array}\right]
\end{aligned}
$$


Where $U\left(\theta_{k i}^{t+1}\right)$ is Quantum rotating gate, $\theta_{k i}^{t+1}$ is the $\mathrm{t}+1$ loop quantum rotation angle. If $\theta_{k i}^{t+1}=0$, then the qubit $W_{k i}$ by quantum non gate $\bar{N}$ to update, the mathematical formula for

$$
W_{k i}^{t+1}=\bar{N} W_{k i}^{t}=\left[\begin{array}{ll}
0 & 1 \\
1 & 0
\end{array}\right] W_{k i}^{t}
$$

\subsection{The Principle of Quantum Artificial Bee Colony Algorithm}

Quantum Swarm consists of two sub groups: quantum observe bee and quantum worker bee. Quantum bee locations are mapped by a food source consisting of 0 or 1 digit coded digits, the definition of the $k$-th location of food sources as $x_{k}=\left(x_{k 1}, x_{k 2}, \mathrm{~L}, x_{k l}\right)$.

$$
x_{k d}^{t+1}=\left\{\begin{array}{l}
1, \eta_{k d}^{t+1}>\left(\alpha_{k d}^{t+1}\right)^{2} \\
0, \eta_{k d}^{t+1} \leq\left(\alpha_{k d}^{t+1}\right)^{2}
\end{array}\right.
$$

Where $d=1,2,3, \ldots l, \quad \eta_{k d}^{t+1} \in[0,1], \quad\left(\alpha_{k d}^{t+1}\right)^{2}$ is the probability of appear 0 with the quantum bit $x_{k d}^{t+1}$.

The definition of local best position is expressed as $p_{g}=\left(p_{g 1}, p_{g 2}, p_{g 3}, \mathrm{~L}, p_{g l}\right)$, the global best bees of $p_{k}=\left(p_{k 1}, p_{k 2}, p_{k 3}, \mathrm{~L}, p_{k l}\right)$.

Quantum bees mainly based on the current location and search for local optimum global optimum colony guide to update the local optimal location, update formula is

$$
\begin{gathered}
\theta_{i d}^{t+1}=e_{1}\left(p_{i d}^{t}-x_{i d}^{t}\right)+e_{2}\left(p_{g d}^{t}-x_{i d}^{t}\right) \\
W_{k i}^{t+1}=\left\{\begin{array}{l}
\bar{N} W_{i d}^{t}, \text { if } \theta_{i d}^{t+1}=0, \text { and } \gamma_{i d}^{t+1}<c_{1} \\
a b s\left[U\left(\theta_{i d}^{t+1}\right) W_{i d}^{t}\right], \text { otherwise }
\end{array}\right.
\end{gathered}
$$

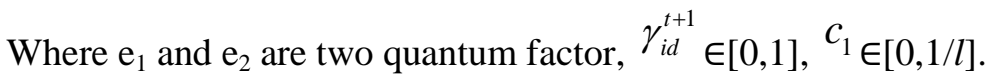

Quantum observation of bee location update, generally choose the other a good quantum worker, through the excellent worker of local optimal position boot, then the $\mathrm{i}$-th only quantum worker is selected probability is

$$
q_{i}^{t+1}=\frac{f\left(p_{i}^{t}\right)}{\sum_{k=1}^{h / 2} f\left(p_{i}^{t}\right)}
$$

$f\left(p_{i}^{t}\right)$ is the i-th only quantum workers local optimal location of adaptation degree function, also is the optimization target function of reciprocal. In addition, the evolution of quantum observation bee is expressed as a mathematical formula

$$
\begin{gathered}
\theta_{j d}^{t+1}=e_{3}\left(p_{i d}^{t}-x_{j d}^{t}\right)+e_{4}\left(p_{j d}^{t}-x_{j d}^{t}\right)+e_{5}\left(p_{g d}^{t}-x_{j d}^{t}\right) \\
W_{i d}^{t+1}=\left\{\begin{array}{l}
\bar{N} W_{j d}^{t}, \text { if } \theta_{j d}^{t+1}=0, \text { and } \gamma_{j d}^{t+1}<c_{2} \\
a b s\left[U\left(\theta_{j d}^{t+1}\right) W_{j d}^{t}\right], \text { otherwise }
\end{array}\right.
\end{gathered}
$$


Where $j=h / 2+1, h / 2+2, \ldots, h, e_{3}, e_{4}$ and $e_{5}$ are three influencing factors. $\gamma_{j d}^{t+1} \in$ $[0,1] . c_{1} \in[0,1 / l]$.

\section{Application of QABC in WSNs}

This paper introduced the QABC into wireless sensor network clustering algorithm. Compared with the classical clustering LEACH algorithm, the biggest difference lies in the different ways of selecting the cluster heads. When selecting the cluster head, the algorithm in this paper considered the distance between the nodes, residual energy, network architecture and the distance from the node to the cluster head etc. and then applied the QABC algorithm to determine the optimal solution as the cluster head node in every round and section. WSNs clustering algorithm can be divided into four phases including initialization phase, clustering phase, stabilizing phase and renewal phase.

1. Initialization phase. After the nodes in the network completed the employment, the nodes will inform the neighboring nodes via broadcast. After the neighboring nodes received the signal, we can calculate the distance between the neighboring nodes to the Sink nodes. After that, the node will send the obtained value and the residual energy to the Sink. The Sink will store the received data in the storage area via data sheet.

2. Clustering phase. The task of clustering phase is to select the cluster head node and then form the cluster small area. The cluster head selection is accomplished in the network base station. The feasible solution of the optimized problems shall be ascertained before the quantum artificial bee colony algorithm. The feasible solution of the optimization function will select in the candidate cluster head node sequence. Only the residual energy of the nodes meets certain conditions, the node will be in addition to the candidate node sequence. After selecting the cluster head, the node in the corresponding area will conduct the configuration according to the received command.

3. Stabilizing phase. After the completion of the network construction in the whole clustering section, the nodes in the cluster will start to transmit the data. The normal cluster nodes will send the gathered information to the cluster head according to the TDMA method and then the cluster head will send the received data to the Sink after integration.

4. Renewal phase. Due to the limited node energy, the selected cluster head will consume large energy after the specified polling times. Hence, the network shall be refreshed. In the renewal phase, after the cluster head sends the gathered information and residual energy to the Sink node, the Sink node will update the residual energy of a node in the storage data sheet. After that, we started from the clustering phase to reselect the cluster head over and over again until all the node energy had been consumed.

\section{Routing Algorithm of Reliability Evaluation Indicators}

There are numerous evaluation indicators for wireless sensor network routing algorithm. This paper mainly conducted the algorithm evaluation the following five aspects: network energy consumption balance analysis, load balance analysis, total energy consumption, amount of survival nodes and network reliability.

(1) Energy consumption balance analysis

Wireless sensor network is mostly characterized by the data-centered to send the data gathered by the sensor node to the Sink. The heavy tasks of the neighboring nodes of Sink will easily cause the network congestion or even lead to the energy hole problem. The unbalanced network energy consumption load will affect the network lifetime. The energy consumption balance is an important parameter for the network performance optimization and the key factor to prolong the network lifetime. In general, energy consumption 
balance analysis will be expressed by the variance of all the node loads. Mathematical formula is expressed as

$$
\varphi=\frac{\sum_{i=1}^{n}\left(E_{i}-\bar{E}\right)^{2}}{n}
$$

Where $E_{i}$ is the energy consumption of the sensor node, $\bar{E}$ is the average of the network load, and $n$ is the number of nodes of WSNs. The bigger the load variance $\varphi$ value of the node, the worse the load balance of the load, and if the smaller the $\varphi_{\text {value, }}$ the better the network energy balance.

(2) Load balancing analysis

The load balancing factor (LBF) is intended to wireless sensor network architecture. A fast and efficient evaluation strategy shall be provided aiming at the network performance analysis to improve the network throughput, data processing capacity, network efficiency and prolong the network lifetime. The node degree is related to the load balancing factor. The node degree usually refers to the number of neighboring nodes. The node degree will reflect the network connected state. The larger $L_{L B F}$ means better network load balance performance, vice versa. Mathematical formula is expressed as

$$
L_{L B F}=\frac{n_{c}}{\sum_{i=1}^{n_{c}}\left(x_{i}-u\right)^{2}}
$$

Where $n_{c}$ is the number of nodes in the sensor network, $x_{i}$ is the number of $\mathrm{i}$-th nodes in the cluster head, and $u$ is the average node number of all the head nodes of the cluster.

(3) Network reliability analysis

The reliability of wireless sensor network refers to the probability of one path existed between the given sending node and goal node, namely the communication probability between two nodes. The normal communication requires the nodes to be in an efficient condition. Under the given working condition and time quantum $\left(t_{1}, t_{2}\right)$, the reliability of wireless sensor network means that the performance of the object at the whole time frame is better than the probability required by the lowest performance in this condition.

$$
R_{w}\left(t_{1}, t_{2}\right)=P\left(f(t) \geq f_{w} \mid t_{1}<t<t_{2}\right)
$$

Here $f(t)$ represents the reliability of the $t$ moments, while the $f_{w}$ represents the worst feature values for the $W$, which is more than $f_{w}$ that the network is reliable.

\section{Algorithm Comparison and Performance Evaluation}

The simulation environment is set to the monitoring area $100 \mathrm{~m} \times 100 \mathrm{~m}$ and random spread 100 nodes in a wireless sensor network, the whole simulation runs time as the 1500s. Experimental simulation computer configuration for $4 \mathrm{G}$ memory of the Pentium processor, the simulation environment is used Matlab, version R2008a.The energy consumption parameters are required for transmitting and receiving in the network wireless communication is set to $E_{T x}=E_{R x}=E_{\text {elec }}=50 \mathrm{~nJ} / \mathrm{bit}, E_{\text {amp }}=100 \mathrm{pJ} / \mathrm{bit} / \mathrm{m}^{2}$. Quantum artificial bee colony parameter settings: population size is 80 , the number of cycles $\mathrm{C}=$ $1000, \mathrm{e}_{1}=0.07, \mathrm{e}_{2}=0.03, \mathrm{e}_{3}=0.02, \mathrm{e}_{4}=0.05, \mathrm{e}_{5}=0.03, \mathrm{c}_{1}=\mathrm{c}_{2}=0.2 / l$, leading bee and follow the total number of bee $\mathrm{S}$ are 50, and the total number of each half, the control parameters define the number 10 , bees initial weights $r_{\min }=0.4, r_{\max }=1.2, r$ initial value is set to 1 , the threshold $\varepsilon=10-6$. The parameters used in the experiment are given in Table 1 . 
Table 1. Simulation Experiment Parameter Settings

\begin{tabular}{l|l}
\hline Parameter & Value \\
\hline Network size & $100 \mathrm{~m} \times 100 \mathrm{~m}$ \\
Number of nodes & 100 \\
Initial energy & $0.5 \mathrm{~J}$ \\
Range & $10 \mathrm{~m}$ \\
ETX & $50 \mathrm{~nJ} / \mathrm{bit}$ \\
ERX & $50 \mathrm{~nJ} / \mathrm{bit}$ \\
Packet & $4 \mathrm{~kb}$ \\
Times & $1500 \mathrm{~s}$ \\
\hline
\end{tabular}

In this paper, we mainly on the following aspects of the simulation experiments: energy balance analysis, load balance analysis, the total energy consumption of the network, node survival and network reliability. Compared with clustering algorithm LEACH [12], HEED primary and secondary clustering algorithm [13], artificial bee colony clustering algorithm [14] and clustering algorithm proposed in this paper.

(1) Energy consumption balance

As an important parameter factor to evaluate the network lifetime, network energy consumption analysis reflects the important indicator of network performance. The simulation results of network energy consumption balance of LEACH clustering algorithm, HEED clustering algorithm, artificial bee colony clustering algorithm and the clustering algorithm in this paper. The simulation results are showed in Figure 3.

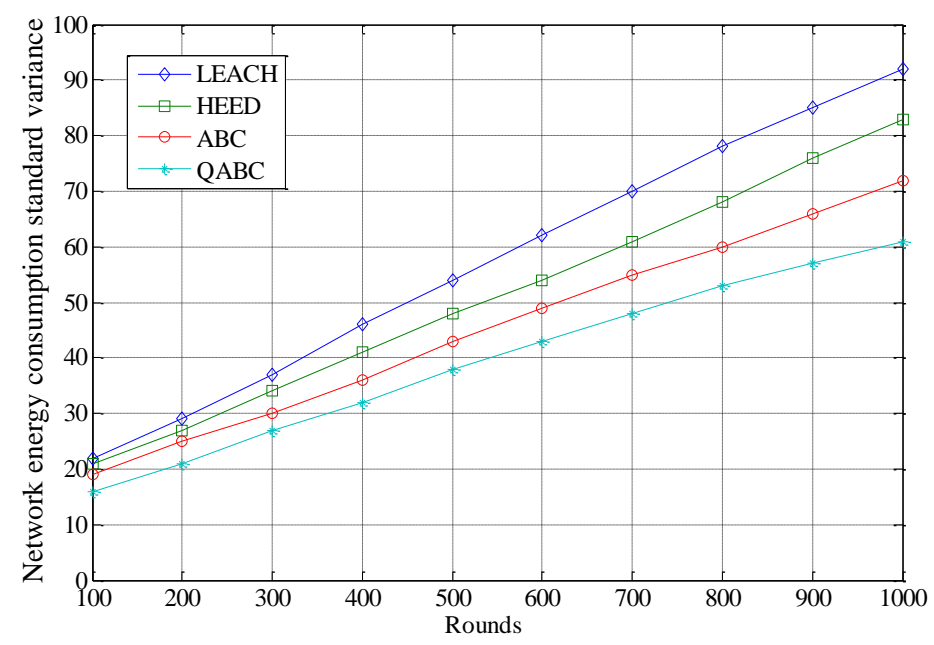

Figure 2. Comparison of Energy Consumption Balanced

It can be seen from Figure 3 that with the increasing network polling times, the variance of the network energy consumption of four algorithms was about 20 at the beginning of the simulation. The standard variance of network energy consumption was increasing gradually, in which the variance of network energy consumption of LEACH algorithm increased linearly with the maximum amplification, then came to the HEED algorithm. The standard variance of network energy consumption of bee colony owned rather small amplification. The algorithm proposed in this paper had the minimum amplification, which indicated that the algorithm in this paper owns the best network energy consumption balance. 
(2) Load balance analysis

The load balance is an important basis to evaluate the network performance, because the load balance can prolong the network lifetime and improve the network efficiency. The simulation results of network load balance of four algorithms are showed in Figure 4.

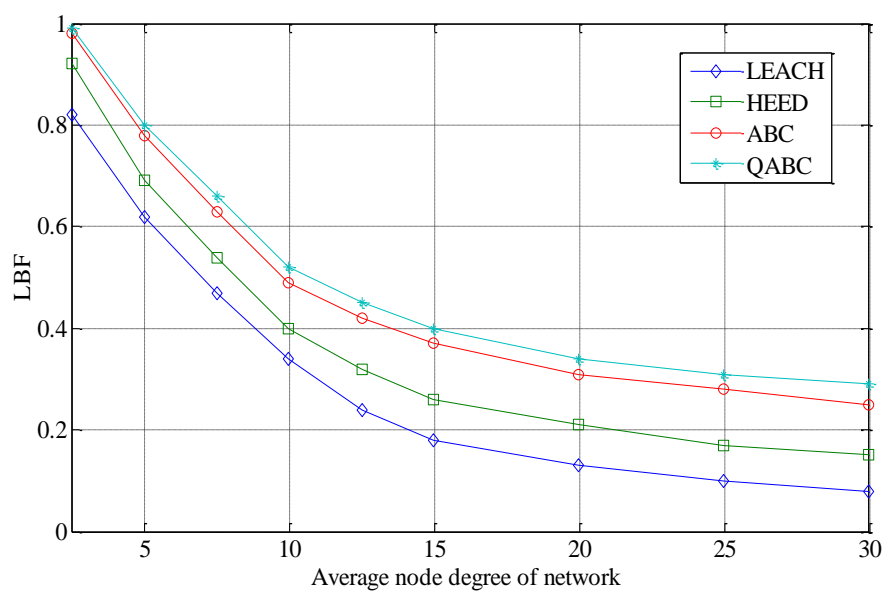

Figure 2. Comparison of Network of LBF

It can be seen from Figure 4 that network load balance factor $L_{L B F}$ will decrease with the increasing average node degree with the non-linear relationship. The LEACH algorithm owned the maximum decreasing amount, which indicated that this algorithm shared worse load balance performance than the other three algorithms with the corresponding short network lifetimes that can be verified by the total energy consumption experiment. Compared with the LEACH algorithm, the HEED algorithm had better load balance. The ABC algorithm was better than the HEED algorithm. However, the algorithm proposed in this paper owed the best load balance factor effect.

(3) Total energy consumption

The total energy consumption is also an important indicator to evaluate the network lifetime, network performance and reliability in wireless sensor network. The comparison of total energy consumption of four algorithms is showed in Figure 5.

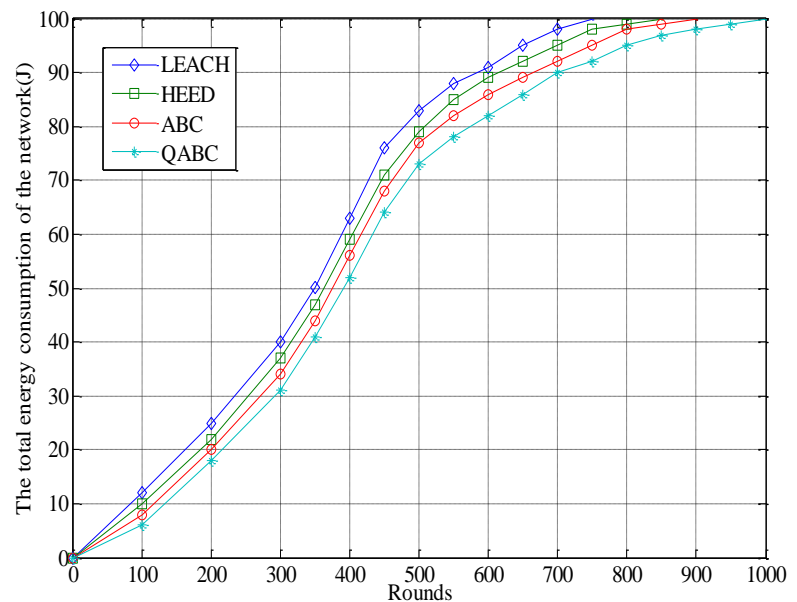

Figure 5. Consumption of Network Energy Consumption

It can be seen from Figure 5 that with the increasing network polling times, the network energy consumption is also increasing gradually, of which the network energy of LEACH algorithm will be used up with about 800 polling times, while the network 
energy of HEED algorithm will be used up with 850 polling times with the ABC algorithm of 930 polling times and the algorithm in this paper of about 1000 polling times. Therefore, the network lifetime of four algorithms is ranked from the LEACH algorithm, HEED algorithm and $\mathrm{ABC}$ algorithm and then the algorithm proposed in this paper.

(4) Amount of survival nodes

We can define the amount of network survival nodes as the process that from the construction, all the nodes in the network worked out of consumption until "death" then network survival nodes are all "dead", of which the network life cycle refers to the whole period from the network operation until the nodes in the whole network are "dead" out of the consumption. Figure 6 provided the comparison of the amount of network survival nodes in the network life cycle of four algorithms.

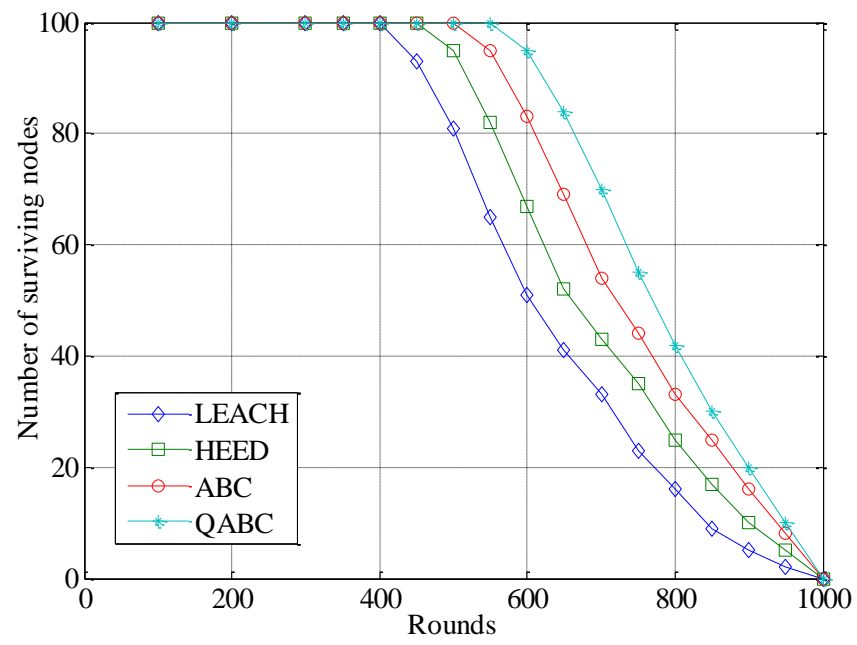

Figure 6. Comparison of the Number of Network Nodes Alive

It can be seen from Figure 6 that at the initial stage of the simulation, due to the enough energy, the network nodes is working regularly. With the simulation, after the 400 polling times, the amount of network survival nodes of four algorithms is decreasing gradually. The amount of network nodes of LEACH algorithm is decreasing gradually at the time of 400 polling times. The amount of network survival nodes of HEED algorithm will be decreasing gradually at the time of 430 polling times with the $\mathrm{ABC}$ algorithm at the time of 510 polling times. The amount of survival nodes of the algorithm in this paper will decrease gradually at the time of 550 polling times. The following decreasing amount is ranked from the LEACH algorithm, HEED algorithm, ABC algorithm and then the algorithm proposed in this paper.

(5) Network reliability

The limited resources, badly applied environment, rapid energy consumption and the inference of the external environment of wireless sensor network caused the breakdown or even collapse of the whole network. The network reliability is a very important evaluation indicator for the network performance. The variation tendency of wireless sensor network reliability of four algorithms with the network polling times is showed in Figure 7. 


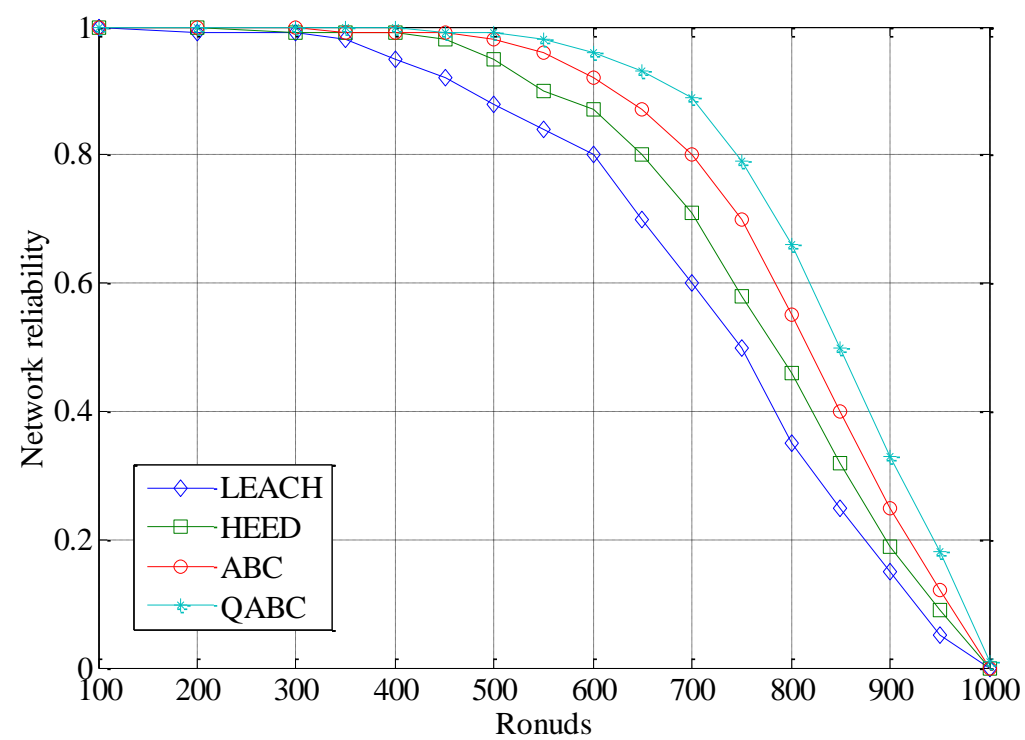

Figure 7. Comparison of Network Reliability

It can be seen from Figure 7 that the network reliability of different algorithms varied from the changing of time polling. Reliability of LEACH algorithm will reduce with the polling times of about 350, while the HEED algorithm will reduce with the polling times of 400 times with the ABC algorithm of 470 times. Network reliability of the algorithm in this paper will decrease gradually with the polling times of 500 times. The decreasing amount is ranked from LEACH algorithm, HEED algorithm, ABC algorithm and then the algorithm in this paper. Hence, the algorithm put forward in this paper own the best network reliability.

It can be observed from the above simulation experiments, we proposed the algorithm in this paper owned efficient routing performance and relatively well reliability but with long time on network simulation. In order to compare the simulation time of four algorithms, this paper conducted 100 times of simulation experiment repeatedly to calculate the average time of network simulation experiment. The average consumed time of four algorithms is showed in Table 2.

Table 2. Comparison of Simulation Time with Various Algorithms

\begin{tabular}{lc}
\hline Algorithm & Run time(S) \\
\hline LEACH & 8.61 \\
HEED & 2.84 \\
ABC & 7.05 \\
QABC & 5.67 \\
\hline
\end{tabular}

It can be seen from Table 2 that it is still acceptable though with the prolonged simulation time. The optimized routing algorithm proposed in this paper owns relatively high network efficiency and reliability, which improves the working efficiency of wireless sensor network, reduces the energy consumption and prolongs the network life cycle.

\section{Conclusion and Future Work}

This paper presented an efficient and reliable WSNs clustering algorithm based on the quantum artificial bee colony algorithm aiming at the unbalanced load in the process of wireless sensor network clustering without considering the residual energy of node, node position and node intensity etc.. This algorithm can better adapt to the network topology, 
evaluate the balanced distribution of network node and guarantee that every round can select the cluster head with the proper ratio, reduces the node energy consumption and prolongs the network lifetime. The experimental results indicated that this algorithm can guarantee the network connected reliability and improve the network efficiency to some extent with relatively good stability and anti-interference. The next step is to apply the quantum artificial bee colony algorithm into the network clustering and data acquisition of mobile wireless sensor network.

\section{Acknowledgments}

The authors would like to thank the anonymous reviewers for helpful comments which helped them improve the technical quality of the paper. This study was supported by Henan Science and Technology Department of Basic and advanced technology research projects (132300410276, 142300410339).

\section{References}

[1] Y.-H. Lin, S.-Y. Chang and H.-M. Sun, "CDAMA: Concealed Data Aggregation Scheme for Multiple Applications in Wireless Sensor Networks", Knowledge and Data Engineering, IEEE Transactions, vol. 25, no. 7, (2013), pp. 1471-1483.

[1] H.-Y. Fu, Y.-J. Pan, Y.-C. Wu and Z. Hu, "An effective routing of large-scale wireless sensor networks for event monitoring", Journal of Computational Information Systems, vol. 9, no. 23, (2013), pp. 9583-9592.

[2] J.-Y. Heo, J.-M. Hong and Y.-K. Cho, "EARQ: Energy Aware Routing for Real-Time and Reliable Communication in Wireless Industrial Sensor Networks", IEEE Transactions on Industrial Informatics, vol. 5, no. 1, (2009), pp. 3-11,

[3] D. C. Hoang; P. Yadav, R. Kumar and S. K. Panda, "Real-Time Implementation of a Harmony Search Algorithm-Based Clustering Protocol for Energy-Efficient Wireless Sensor Networks", IEEE Transactions on Industrial Informatics, vol. 10, no. 1, (2014), pp. 774-783.

[4] J.-B. Hou, X.-G. Fan, W.-L. Wang, J. Jie and Y. Wang, "Clustering strategy of Wireless Sensor Networks based on improved Discrete Particle Swarm Optimization", Natural Computation (ICNC), Sixth International Conference, (2010).

[5] Z.-P. Gao, D.-P. Liu, Y. Yang, J.-C. Zheng and Y.-W. Hao, "A load balance algorithm based on nodes performance in Hadoop cluster", Network Operations and Management Symposium (APNOMS), 16th Asia-Pacific, (2014), pp.1-4.

[6] Y.-S. Jiang, W.-R. Shi and X.-G. Wang, "A distributed routing for wireless sensor networks with mobile sink based on the greedy embedding", Ad Hoc Networks, vol. 20, (2014), pp. 150-162.

[7] J.-S. Leu, T.-H. Chiang, M.-C. Yu and K.-W. Su, "Energy Efficient Clustering Scheme for Prolonging the Lifetime of Wireless Sensor Network With Isolated Nodes", Communications Letters, IEEE , vol.19, no.2, (2015), pp. 259-262.

[8] S. Ganesh and R. Amutha, "Efficient and secure routing protocol for wireless sensor networks through SNR based dynamic clustering mechanisms", Communications and Networks, Journal, vol. 15, no. 4, (2013), pp. 422-429.

[9] Y. Liu, X.-X. Ling, Y. Liang and G.-H. Liu, "Improved artificial bee colony algorithm with mutual learning", Systems Engineering and Electronics, Journal, vol. 23, no. 2, (2012), pp. 265-275.

[10] C.-C. Hung, E. Casper, B.-C. Kuo, W.-P. Liu; E. Jung and M. Yang, "A Quantum-Modeled Artificial Bee Colony clustering algorithm for remotely sensed multi-band image segmentation", Geoscience and Remote Sensing Symposium (IGARSS), IEEE International, (2013).

[11] Z.-C. Zhang and X.-X. Zhang, "Research of improved clustering routing algorithm based on load balance in wireless sensor networks", Wireless Mobile and Computing (CCWMC), IET International Communication Conference, (2009).

[12] J. Y. Lee, K. D. Jung, B. Shrestha, J. Lee and S. Cho, "Energy efficiency improvement of the of a cluster head selection for wireless sensor networks", Int. Journal of Smart Home, vol. 8, no. 3, (2014), pp. 9-18.

[13] S. Okdem, D. Karaboga and C. Ozturk, "An application of Wireless Sensor Network routing based on Artificial Bee Colony Algorithm", Evolutionary Computation (CEC), IEEE Congress, (2011), pp. 326330. 


\section{Authors}

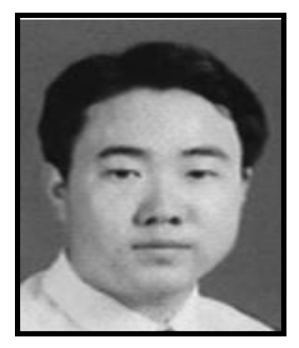

Huadong Wang, received the master's degree in computer science and technology from Zhengzhou University, China in 2008. Currently, he is a researcher at Zhoukou Normal University, China. His research interests include Computer Applications and Computer Network.

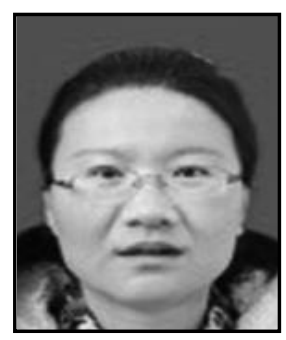

Dayu Wang, received the bachelor's degree in computer science and technology from Henan University, China in 2012. Currently, she is a researcher at Zhoukou Normal University, China. She research interests include Computer Applications and Computer Network. 
International Journal of Control and Automation Vol.10, No.3 (2017) 